\title{
Pseudoaneurysm of Splenic Artery - A Severe Vascular Complication of Pancreatitis
}

\author{
Varun Singh ${ }^{1}$, Suresh V Phatak ${ }^{2}$, Shirish Vaidya ${ }^{3}$, Rajasbala P Dhande ${ }^{4}$, Shreya Tapadia ${ }^{5}$ \\ 1, 2, 3,4,5 Department of Radio Diagnosis, Jawaharlal Nehru Medical College, \\ DMIMS, Sawangi, Wardha, Maharashtra, India.
}

\section{INTRODUCTION}

Acute pancreatitis is one of the common abdominal pathologies having an incidence of 10 to 100 cases per 100,000 population worldwide with varying type of presentation ranging from self-limiting abdominal pain to high mortality and morbidity due to organ failure. The major risk factors leading to pancreatitis include gall stones and alcohol consumption, others being trauma, drugs, and infections. ${ }^{1,2}$ The revised Atlanta classification (2012) has classified acute pancreatitis on the basis of local, systemic complications and organ failure as mild, moderately severe and severe type of pancreatitis. One of the dreaded local complications of pancreatitis are the vascular complications occurring with a frequency of $1.2-14 \%$, with a greater incidence seen in chronic pancreatitis (7-10\%) than acute pancreatitis $(1-6 \%)$. The two main types of vascular complications being splenic vein thrombosis (more common) and pseudo aneurysm of (in order of decreasing frequency) the splenic, gastro duodenal, and pancreaticoduodenal arteries.3,4 The common symptoms of pseudoaneurysm of splenic artery are abdominal pain, hematemesis, melena, flank pain, and chest pain. Haemorrhage from pseudo aneurysm can be found in the pseudo cyst, stomach, pancreatic duct, peritoneal cavity, or retro peritoneum making the patient highly haemodynamically unstable at presentation having high rates of morbidity and mortality. 5 The mortality rate due to haemorrhages directly related to the pancreas is up to $50 \%{ }^{2}$ Clinical diagnosis in these patients is highly difficult due to varied range of symptoms and hence ultrasound coupled with contrast enhanced CT imaging helps in accurate diagnosing of such an entity.

Pancreatitis is one of the common abdominal pathologies having variable type of presentation. Vascular complications are rare, but severe in nature having high mortality and morbidity rates. Ultrasound coupled with computer tomography helps in fast and accurate diagnosis of these vascular complications like pseudo aneurysms, aneurysms and thrombosis of peri pancreatic arteries. We are presenting a case report of pseudoaneurysm of splenic artery.

\section{PRESENTATION OF CASE}

A 33 yrs. old alcoholic male patient with a known history of pancreatitis came to the emergency department with a history of severe abdominal pain and hematemesis since last 7 days. On clinical examination the patient was afebrile, had distension and tenderness of abdomen. Lab investigation suggested raised lipase (500 IU / L) and amylase (1100 IU / L) levels.

The patient was then referred to The Department of Radiodiagnosis, the first line of investigation conducted was an ultrasound which revealed the pancreas to be atrophic with multiple parenchymal calcifications in the head region. (Figure 1) and there is e / o a well-defined, heterogeneous round to oval lesion in the region of splenic hilum, having an eccentric hypo echoic lumen, with surrounding thrombus. On colour Doppler, the lumen showed characteristic yin yang sign indicating bidirectional flow due to the swirling of blood within the false aneurysm. (Figure 2, 3). This was followed by contrast enhanced CT of abdomen for further correlation which revealed the pancreas to be atrophic with prominent main pancreatic duct and a calcific focus of $3.5 \mathrm{~mm}$ size noted at the head of pancreas. The splenic artery appeared dilated with a well-defined round lesion of size $5.4 \times 4.5 \mathrm{~cm}$ abutting splenic artery in the tail region of pancreas.
Corresponding Author: Dr. Suresh Phatak.

Radio Diagnosis, Jawaharlal Nehru Medical College, DMIMS, Sawangi, Wardha -

442001, Maharashtra, India.

E-mail: suresh_phatak@yahoo.com

DOI: $10.14260 / \mathrm{jemds} / 2020 / 694$

How to Cite This Article:

Singh V, Phatak SV, Vaidya S. Pseudoaneurysm of splenic artery - a severe vascular complication of pancreatitis. $J$ Evolution Med Dent Sci 2020;9(42):31693171, DOI: 10.14260/jemds/2020/694

Submission 15-07-2020,

Peer Review 08-09-2020,

Acceptance 15-09-2020,

Published 19-10-2020.

Copyright (C) 2020 Varun Singh et al. This is an open access article distributed under Creative Commons Attribution License [Attribution 4.0 International (CC BY 4.0)] 
The lesion shows eccentric hyper dense area of $3.2 \times 2.4$ $\mathrm{cm}$ with a density similar to arterial contrast s / o partially thrombosed pseudo aneurysm of splenic artery. Other extra pancreatic findings were enlarged spleen with multiple periportal, peri-splenic, peri-pancreatic, mesenteric collaterals and ascites.

After confirming the diagnosis of splenic artery pseudoaneurysm the patient underwent endovascular management procedure, where the splenic artery aneurysm was embolised with $3^{\text {rd }}$ generation detachable Guglielmi coils using the sandwich technique.

\section{DISCUSSION}

The major differential diagnosis of pseudoaneurysm is true aneurysm which differs in clinical presentation, pathophysiology and imaging. In true aneurysm all three layers, tunica adventitia, media and intima are affected whereas in pseudoaneurysm only the tunica media and intima are affected. Patients of pseudoaneurysm of splenic artery almost always are symptomatic, having much higher risk of mortality due to the rupture of aneurysm compared to true aneurysms. ${ }^{6}$ The various etiological factors leading to true aneurysm of splenic artery are hypertension, liver transplantation, portal hypertension, cirrhosis and pregnancy whereas pseudoaneurysm are caused by pancreatitis, trauma, iatrogenic and postoperative causes, and, rarely peptic ulcer disease. The incidence of pseudoaneurysm is more common in females compared to males by four times. ${ }^{7,8}$

On grayscale, a patent pseudo aneurysm appears as an anechoic round or ovoid structure. Similar appearance is seen in other fluid collections, including cysts, seromas, or haematomas, hence, colour Doppler imaging is used to confirm the presence of blood flow within the pseudo aneurysm which shows the characteristic Ying Yang sign, indicating the to and fro movement of blood across the neck of the aneurysm. When present, thrombus in the pseudo aneurysm appears mildly echogenic or hypo echoic without flow; it may be mural or centrally fill a portion of the pseudo aneurysm lumen. Turbulent blood flow in the pseudoaneurysm is illustrated by interchangeable colouring appearance, either in red or blue colour. A "to-and-fro" pattern at the neck of the lesion is confirmatory of a pseudo aneurysm. ${ }^{9}$

The basic pathophysiology behind pseudoaneurysm is vessel wall injury in cases of severe pancreatic inflammation and necrosis due to the local spread of exocrine proteolytic and lipolytic enzyme-rich fluids. These fluids cause weakening and electrolytic erosions of the vessel wall, which may result in the formation of a pseudo aneurysm. These can be associated with active bleeding or can contain hematoma (if the pseudo aneurysm becomes thrombosed), or can lead to frank intraperitoneal haemorrhage (if the pseudo aneurysm ruptures). 4

Ultrasonography (US) is a widely used, non-invasive imaging modality for the investigation of vascular diseases. The main advantage of US imaging is it being cheap, readily available, non-ionizing in nature and having a sensitivity and specificity of $94 \%$ and $97 \%$ respectively. ${ }^{10}$

Various treatment methods are known for management of splenic artery pseudoaneurysm like the bipolar surgical ligation, aneurysmectomy and interventional percutaneous embolization or stenting. The surgical management is associated with high mortality rates, especially when pseudoaneurysm is associated with pancreatitis (16\%).
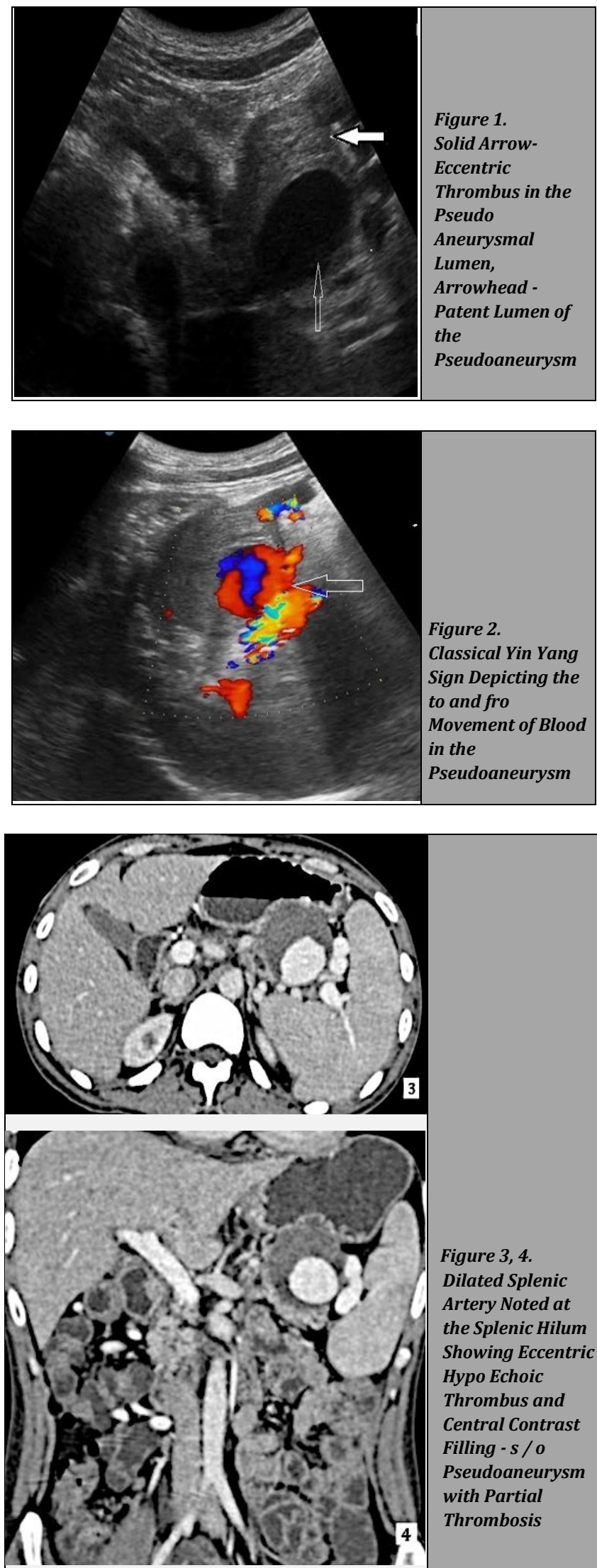

In today's time and age the most popular mode of managing cases of pseudo and true aneurysm of splenic artery is by packing the aneurysmal sac with embolic agents like $3^{\text {rd }}$ 
generation detachable Guglielmi coils or stent grafts using the sandwich technique, where a balloon catheter is inflated across the neck of the aneurysm to keep the coils in their place. ${ }^{11}$

To conclude USG, Doppler and CECT abdomen are excellent modalities for early and accurate diagnosis of pseudoaneurysm, helping clinicians in managing the vascular complications of pancreatitis.

Financial or other competing interests: None.

Disclosure forms provided by the authors are available with the full text of this article at jemds.com.

\section{REFERENCES}

[1] Thoeni RF. The revised Atlanta classification of acute pancreatitis: its importance for the radiologist and its effect on treatment. Radiology 2012; 262(3):751-64.

[2] Głuszek S, Nawacki Ł, Matykiewicz J, et al. Severe vascular complications of acute pancreatitis. Pol Przegl Chir 2015;87(10):485-90.

[3] Foster BR, Jensen KK, Bakis G, et al. Revised Atlanta classification for acute pancreatitis: a pictorial essay. Radiographics 2016;36(3):675-87.

[4] Barge JU, Lopera JE. Vascular complications of pancreatitis: role of interventional therapy. Korean J Radiol 2012;13 Suppl 1(Suppl 1):S45-55.
[5] Chia C, Pandya GJ, Kamalesh A, et al. Splenic artery pseudoaneurysm masquerading as a pancreatic cyst - a diagnostic challenge. Int surg 2015;100(6):1069-71.

[6] Agrawal GA, Johnson PT, Fishman EK. Splenic artery aneurysms and pseudoaneurysms: clinical distinctions and CT appearances. AJR Am J Roentgenol 2007;188(4):992-9.

[7] Abbas MA, Stone WM, Fowl RJ, et al. Splenic artery aneurysms: two decades experience at Mayo clinic. Ann Vasc Surg 2002;16(4):442-9.

[8] Tessier DJ, Stone WM, Fowl RJ, et al. Clinical features and management of splenic artery pseudoaneurysm: case series and cumulative review of literature. J Vasc Surg 2003;38(5):969-74.

[9] Mahmoud MZ, Al-Saadi M, Abuderman A, et al. To-and-fro waveform in the diagnosis of arterial pseudoaneurysms. World J Radiol 2015;7(5):89-99.

[10] Morgan R, Belli AM. Current treatment methods for postcatheterization pseudoaneurysms. J Vasc Inter Radiol 2003;14(6):697-710.

[11] Madoff DC, Denys A, Wallace MJ, et al. Splenic arterial interventions: anatomy, indications, technical considerations and potential complications. Radiographics 2005;25(Suppl 1):S191-S211. 